\title{
Perioperative interstitial high-dose-rate brachytherapy for keloids scar
}

\author{
Victoria Vera Barragán, MD!, Ana Isabel Alonso García, PhD², José Fernández Garcia, PhD², Marta De Juan Marín, MD², \\ Johanna del Carmen Peña Vivas, MD², Germán Juan Rijo, PhD² \\ 'Hospital Universitario Badajoz, Badajoz, Spain, ${ }^{2}$ Hospital Universitario Central de Asturias, Oviedo, Spain
}

\begin{abstract}
Purpose: To evaluate the results of keloids treated with perioperative interstitial high-dose-rate brachytherapy (HDR-BT)

Material and methods: A retrospective review of 61 keloids in 51 patients treated with perioperative interstitial HDR-BT was performed. Keloid lesions were treated with $12 \mathrm{~Gy}$ in 4 fractions within 48 hours. Time between surgery and brachytherapy was less than 90 minutes. Local failure was defined as recurrence of apparent keloid or symptoms in the same place. Median age of patients was 46 years (range, 20-89 years). Keloid scars were located on the chest $(41 \%)$, ear lobe $(21 \%)$, back $(1.6 \%)$, extremities $(11.5 \%)$, and mean keloid size was $6.3 \mathrm{~cm}$.

Results: With a median follow-up of 47.95 months (range, 1-96 months), the recurrence rate was $4.9 \%$, all located on the chest. The multivariate analysis showed no statistically significant relationship between location, scar size, and previously treated lesions with the recurrence rate.

Conclusions: The treatment of keloid scars with perioperative interstitial high-dose-rate brachytherapy achieved excellent results, with a recurrence rate of only $4.9 \%$ and excellent cosmetic outcomes, suggesting that this technique might be one of the best options for treating keloids.

Key words: brachytherapy, keloids, interstitial perioperative radiotherapy.

\section{Purpose}

Keloids are benign cutaneous tumors caused by an accelerated proliferation of fibroblasts, in response to trauma in the skin, either surgically or after an accident. Excessive proliferation of fibroblasts is mediated by macrophages, cytokines, and mainly growth factors, such as transforming growth factor $\beta$ (TGF- $\beta$ ) among others. It is related to genetic predisposition, autosomal dominant inheritance with incomplete penetrance, and single nucleotide polymorphism, especially in some races, such as Afro-Americans [1].

Keloids usually manifest as a reddish tumor that surpasses the surgical scar and rarely disappears spontaneously. They can produce clinical conditions, including pruritus and pain of medium to severe grade. They are more frequent in the Afro-American population and the novo after any skin insult (for instance, skin piercing, surgery) [2].

Since 1909, the role of adjuvant radiotherapy for resected keloids has become interesting due to high-rates of recurrent disease during treatment with surgery alone. There has been an increase in their treatment due to the aesthetic alteration that some of them can cause, being very visible in areas, such as the face $[3,4]$. However, due to their benign nature, keloids are rarely treated, which is the reason there are no consensus guidelines to optimize the management (or no universally accepted standards of care) [5].

Keloid resection as a single-treatment has been demonstrated to have very poor control of the lesion, with recurrence rate up to $80 \%$ or more. Therefore, multiple adjuvant treatments have been proposed aiming at decreasing the recurrence rate, such as topical corticosteroids or injected into the lesion, intra-lesional interferon, continuous pressure on the scar, therapy laser, tacrolimus, flurandrenolide or imiquimod, and radiotherapy [6]. Multiple radiotherapy techniques have been used, with low-energy X-rays, electrons, or iridium-192 $\left({ }^{192} \mathrm{Ir}\right)$ brachytherapy [2]. Irradiation technique with ${ }^{192} \mathrm{Ir}$ brachytherapy as a complementary treatment to surgical excision was originally described by Nicolettis and Chassagne in 1967, being today the most common treatment for keloids [3].

Combining surgery and radiotherapy, administered as perioperative brachytherapy, has been proved to be 
the most effective treatment, achieving a local control above $25 \%$. From a dosimetric point of view, brachytherapy achieves a very focalized and conformal dose to the lesion, thereby reducing the irradiation of healthy tissue in comparison to external radiotherapy [7-9].

In our center, perioperative brachytherapy has been used to treat keloid scars since 2011, accumulating much experience and valuable data. Specifically, since the treatment of malignant tumors is the main activity in most hospitals, collecting a large database of patients treated for benign keloid scars is not straight-forward. The present study aimed to retrospectively review and analyze data from 51 patients in order to find the potential relationship between prognosis or recurrences and long-term cosmesis.

\section{Material and methods}

Inclusion criteria were patients with keloids refractory to at least one topical treatment, with symptoms (pain and pruritus) at the location. Exclusion criteria were pregnant women, individuals under 16, or keloids located in previously irradiated areas.

\section{Patients' characteristics}

From September 2011 to October 2016, a total of 61 keloids in 51 patients were treated. Median age of the patients was 46 years (range, 20-89 years). Twenty-six patients analyzed were men (51\%) and twenty-five were women $(49 \%)$. $71.6 \%$ of patients were Caucasian, $17.9 \%$ were African-American, and $10.5 \%$ were of unknown origin. The reason there is a portion of patients with unknown ethnicity is due to the fact that our center does not

Table 1. Characteristics of keloids under study Characteristics of keloids

\begin{tabular}{lc}
\hline Etiology & \\
\hline Surgical scar & $29 \%$ \\
\hline Traumatism & $32 \%$ \\
\hline Acne & $36 \%$ \\
\hline Others & $3 \%$ \\
\hline Scar size (cm), average (range) & $6.3(2-22)$ \\
\hline Location & \\
\hline Chest & $41 \%$ \\
\hline Ear lobe & $21 \%$ \\
\hline Helix & $11.5 \%$ \\
\hline Back & $1.6 \%$ \\
\hline Extremities & $11.5 \%$ \\
\hline Others & $13.4 \%$ \\
\hline Previous recurrence & \\
\hline Yes & $23 \%$ \\
\hline No & $77 \%$ \\
\hline Ethnicity & $71.6 \%$ \\
\hline Caucasian & $17.9 \%$ \\
\hline Black & $10.5 \%$ \\
\hline Unknown & \\
\hline
\end{tabular}

record the skin color or ethnicity of patients in the clinical history. Being a retrospective database, the ethnicity could only be recorded for those patients who came to the hospital for consultation again, and it remained unknown for those who did not come back.

Average size of the treated scars was $6.3 \mathrm{~cm}$, and percentage of the locations were as follows: the chest $(n=25$, $41 \%)$, earlobe $(n=12,21 \%)$, helix $(n=7,11.5 \%)$, back $(n=2,1.6 \%)$, and extremities $(n=7,11.5 \%)$. Other locations $(n=8,13.4 \%)$ included some less frequent places, as the scalp, face (around cheek region), and one keloid was located sub-umbilically (Table 1).

Before starting the treatment, size and thickness of the scar were analyzed to differentiate between scars below $2 \mathrm{~mm}$ or more. In addition, patients were provided with a questionnaire, with questions on symptoms they presented. Details of the treatment and its' associated possible side effects were also explained to patients. Consent was obtained from all cases.

After the treatment, patient was followed-up through the first month since the end of treatment, and consecutively at 3 , and at 6 months. Subsequently, if there were no side effects that required specific treatment or closer monitoring, follow-up became annual provided by radiation oncology service, alternating with plastic surgery service.

\section{Treatment performed}

All patients were treated with perioperative interstitial high-dose-rate brachytherapy (HDR-BT) using 12 Gy in four fractions, for 48 hours. Interfraction time was 8 hours.

During the procedure, the surgeon made a complete resection of the keloid in all cases. Subsequently, a flexible plastic catheter was placed in surgical wound and a closure without pressure points using non absorbable suture was performed. The catheter for radiation therapy was inserted through the plastic catheter with plastic button. This procedure was performed in the operating room (Figure 1). Time between surgery and first brachytherapy session was less than 90 minutes (range, 23-90 minutes).

The treatment was performed using a radioactive source of cobalt-60 $\left({ }^{60} \mathrm{Co}\right)$. Dosimetry was carried out in depth at $5 \mathrm{~mm}$ from the center of the source, using dosimetric system manually.

A simulation computed tomography (CT) was only used in cases, for which the shape of the scar was complicated, or when there were two keloids nearby (Figure 2). To calculate the plan, a CT scan was performed. Only the target volumes were contoured with a margin around the scar of $0.5 \mathrm{~cm}$, and dosimetric plan was calculated with SagiPlan version 2.1 .

This treatment did not require hospitalization of the patient since the patient is sent home with the plastic catheters in place. The patient was advised to avoid moving the catheters as much as possible, and after last treatment session, the catheter was removed and covered with a dressing after cleaning the scar with povidone iodine.

Cosmetic evaluation was carried out by the same observer, and the doctor arranged new visits. At each one, the observer with the patient evaluated visible or non-vis- 


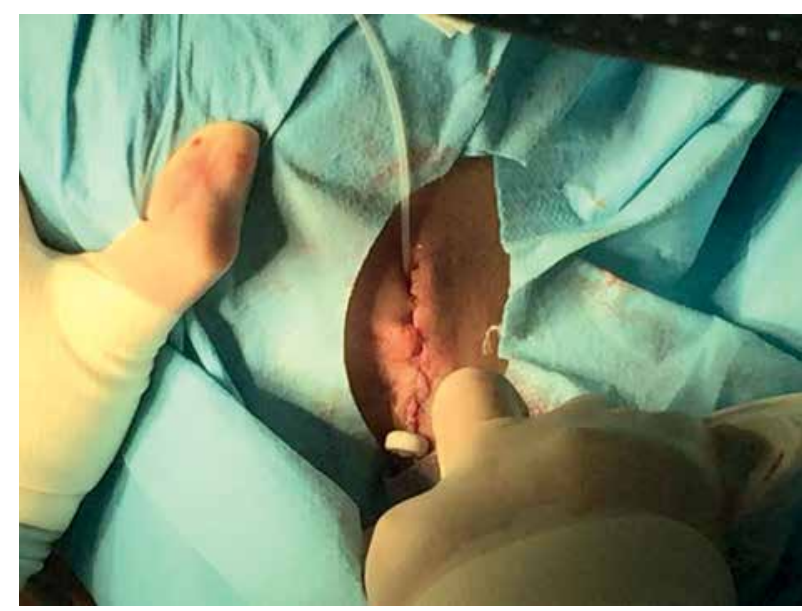

Fig. 1. The surgeon puts the plastic catheter into the wound after resection of the keloid

ible thickening of the scar, and whether or not the symptoms presented before treatment disappeared, with side effects observed in the treated area (telangiectasias or changes in skin color). Local failure was defined as a recurrence of visible keloid, the scar elevating more than $2 \mathrm{~mm}$, and/or vegetation outside border of excision along with local symptoms (itchiness, pain, or infection).

Excellent cosmetic results were reported when there was no thickening of the scar and no side effects (telangiectasias, hypo- or hyperpigmentation of the treated area). Good cosmesis was evaluated as multiple visible side effects in the treatment area, whereas bad cosmesis was considered when there was a thickening of the skin up to $2 \mathrm{~mm}$ above the plane of the skin, or new scarring was deleterious with partial or total hyperplasia (Table 2).

\section{Statistical analysis}

To find correlations between the recurrence rate, different patient, and keloid characteristics, data was analyzed using IBM statistic software v. 24; ANOVA test was applied for quantitative variables (size of the scar) and $X^{2}$ test was used for qualitative variables (ethnicity, location). Statistical relationship was considered significant when $p<0.05$.

\section{BED calculation}

To calculate BED dose, conventional formula [10] was used as follows:

$$
\mathrm{BED}=\mathrm{n} \times \mathrm{d}(1+\mathrm{d} / \mathrm{a} / \beta),
$$

where $n$ is the number of treatment fractions, $d$ is dose per fraction in Gray (Gy), and $(\alpha / \beta)$ is $\alpha / \beta$ ratio. We considered for keloids an $\alpha / \beta$ ratio of 2.08 , according to data supported by Flickinger et al. [11], and $\alpha / \beta=3$ for late skin toxicity. This concept is controversial, and is argued in detail below, in the discussion section.

\section{Results}

With a median follow-up of 50.9 months (range, 16-96 months), the recurrence rate was $4.9 \%$, all located

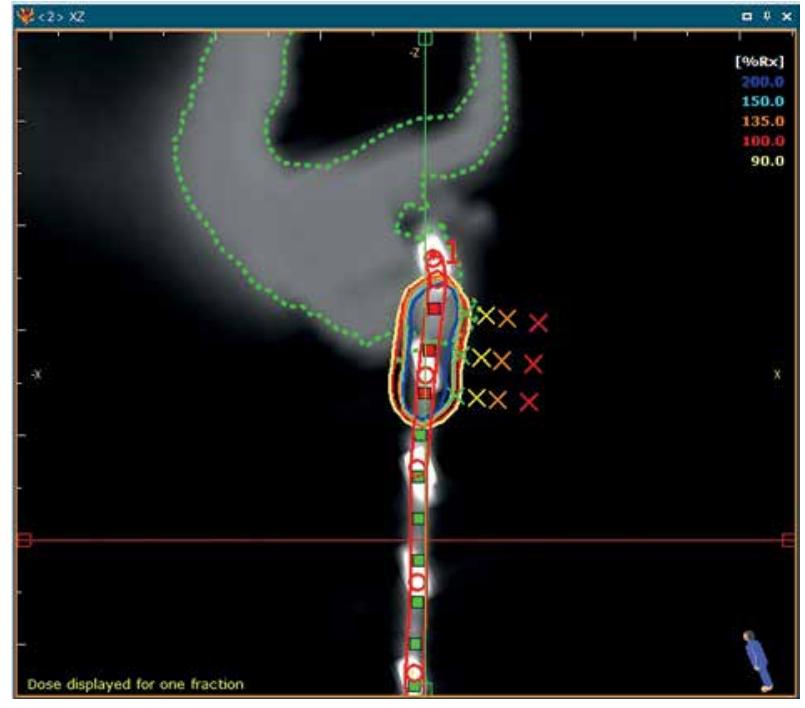

Fig. 2. Treatment plan for a selected patient with a keloid. Lines in the figure represent different isodose regions, and the red line is the CTV

in the thorax. Considering the use of $12 \mathrm{~Gy}$ in 4 fractions and an $\alpha / \beta$ ratio for keloids $=2.08$, biological equivalent dose (BED) was $29.3 \mathrm{~Gy}$ for keloids, and BED was $24 \mathrm{~Gy}$ for healthy tissue late toxicity.

In the analysis of ANOVA and $\chi^{2}$ tests, no statistically significant relationship between scar size and recurrence $(p=0.148)$, or between race and recurrence rate $(p=0.312)$ were observed.

In the sub-group analysis, within the group of keloids located in the thorax, there were $16 \%$ of recurrences, without observing any common characteristics in this group that can explain worst results. Also, no statistical relationship between any specific location and local recurrence was observed $(p=0.09)$. In the set of images, one can notice two different results in two patients under study (Figures 3-7). Figures 8 and 9 present a bad cosmetic result due to secondary telangiectasias along the treatment area. No instance of infection or delayed wound healing due to catheter position were observed in our series. Database of the included 66 keloids evaluated, but 6 patients died and 21 patients did not come back to the hospital for new evaluation. Finally, the cosmetic evaluation was done in 45 keloids treated, and the observed results shown excellent cosmesis in $57 \%$, good in $24 \%$, and poor in $18 \%$ of the keloids.

\section{Discussion}

Keloids result in cosmetic deformities as well as physical symptoms, including itching, stiffness, scar contracture, tenderness, and pain. Effective treatment, such as post-

Table 2. Characteristics of keloids under study

\begin{tabular}{lcc} 
Excellent cosmesis & Good cosmesis & Bad cosmesis \\
\hline No scar thickening & Multiple visible & Scar thickening \\
No side effects & $\begin{array}{c}\text { side effects in the } \\
\text { treatment area }\end{array}$ & present
\end{tabular}




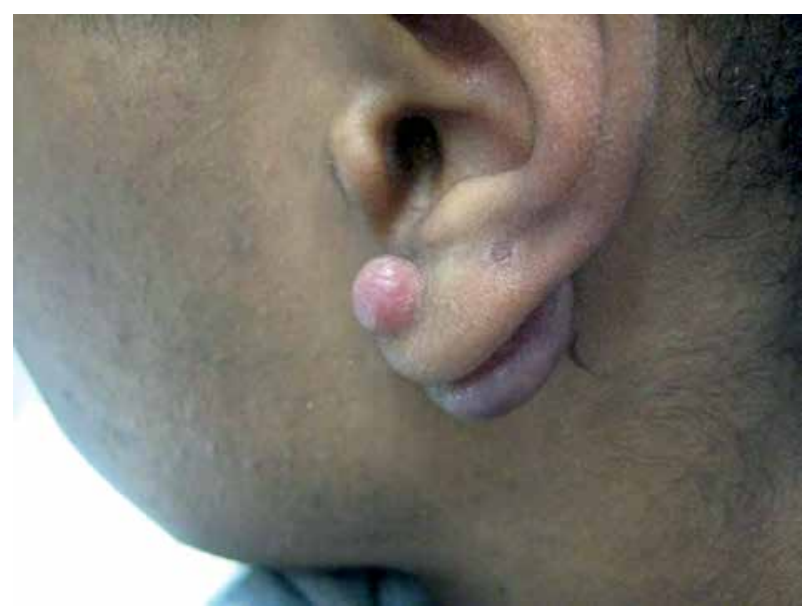

Fig. 3. Ear lobe keloid before the treatment

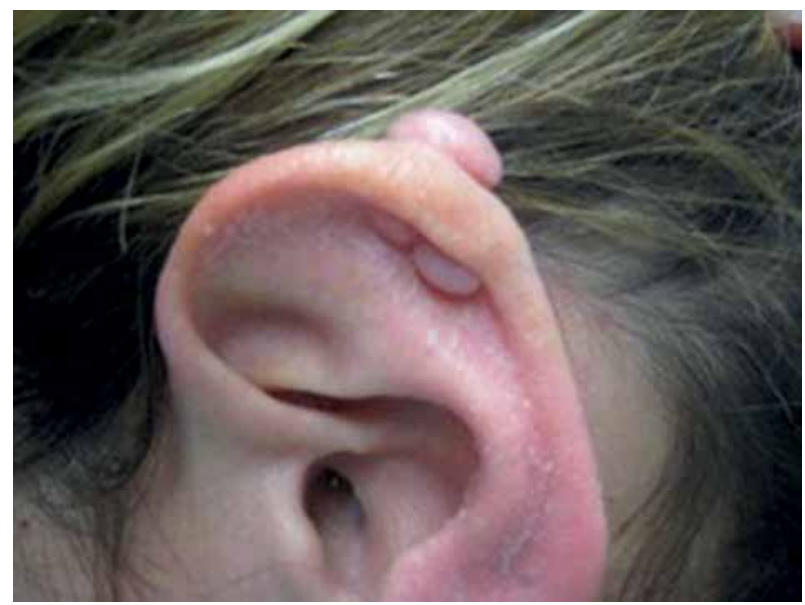

Fig. 5. Ear helix keloid before the treatment

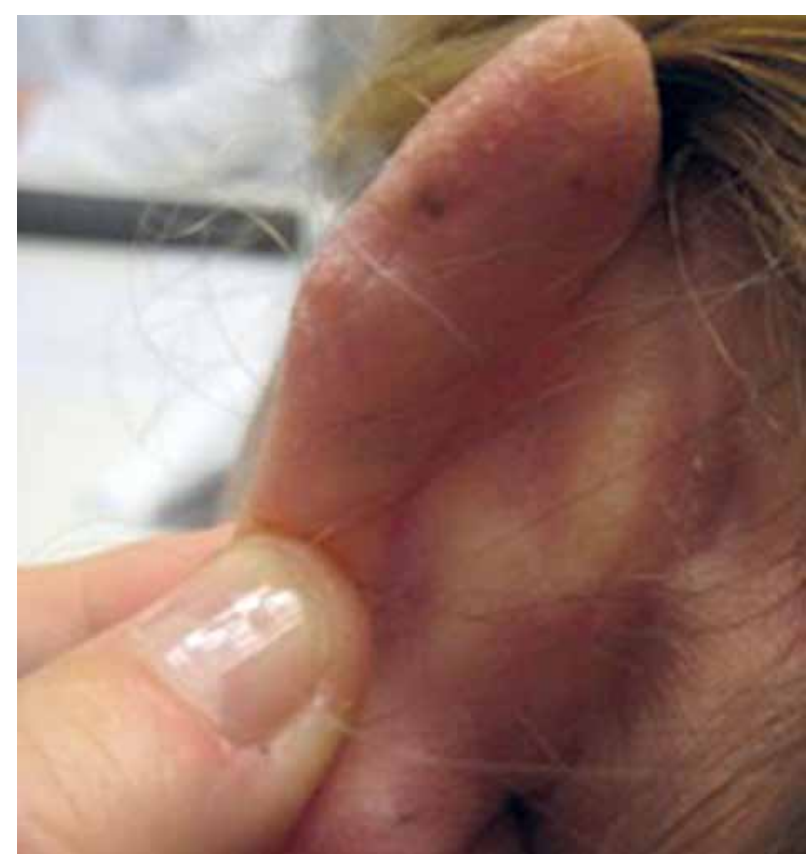

Fig. 7. Ear helix keloid after the treatment

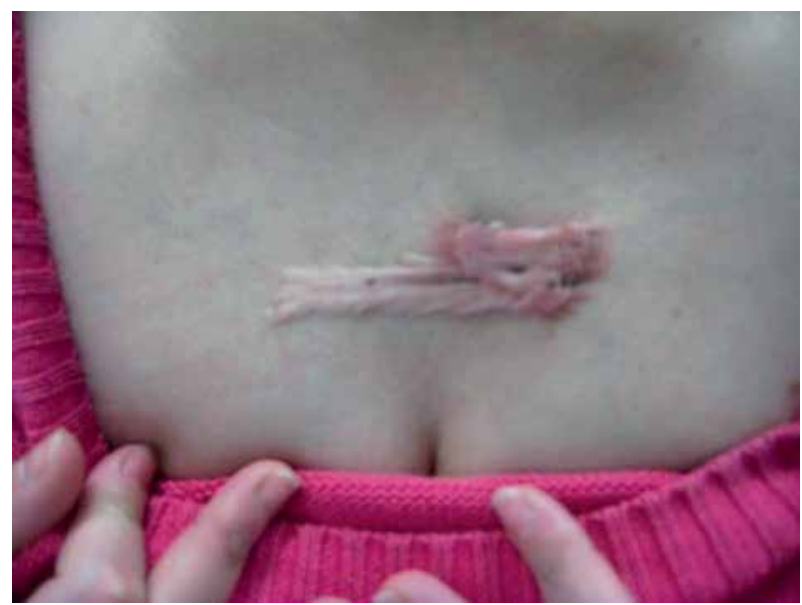

Fig. 4. Pre-sternal keloid treated previously with topical steroids without effect. Status before surgery and brachytherapy treatment

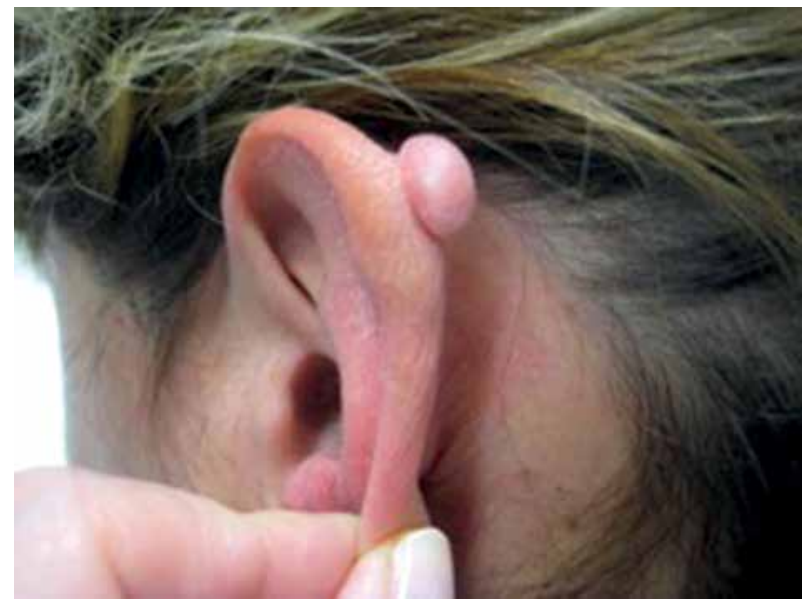

Fig. 6. Ear helix keloid before the treatment

operative radiotherapy, can substantially improve quality of life of patients due to relief in the symptoms [12].

In our series, a recurrence rate of $4.9 \%$ was observed using a dose of 12 Gy in 4 fractions, with a time gap between fractions of 8 hours. The time gap between surgery and first radiotherapy application was less than 90 minutes. Considering an $\alpha / \beta$ ratio for keloids equal to 10 , the results of biological equivalent dose (BED) was 15.6 Gy. However, Veen et al. recommended the use of BED around $30 \mathrm{~Gy}$ to achieve fewer recurrences with that dose level, which would imply that our treatment was below the recommended value [9].

A recent publication with radiobiological analysis of post-operative external radiotherapy from Flickinger et al. [11] supports the hypothesis of a much lower $\alpha / \beta$ ratio for keloids equal to 2.08 . This would suggest that our BED value was higher (around 29.3), which might be more in line with our results, since our local recurrence rate was near to the best data published in the literature.

Previously published data on interstitial brachytherapy is hardly comparable due to variability in dose and implant techniques as well as differences between radiation source and skin distance. However, there are a few 


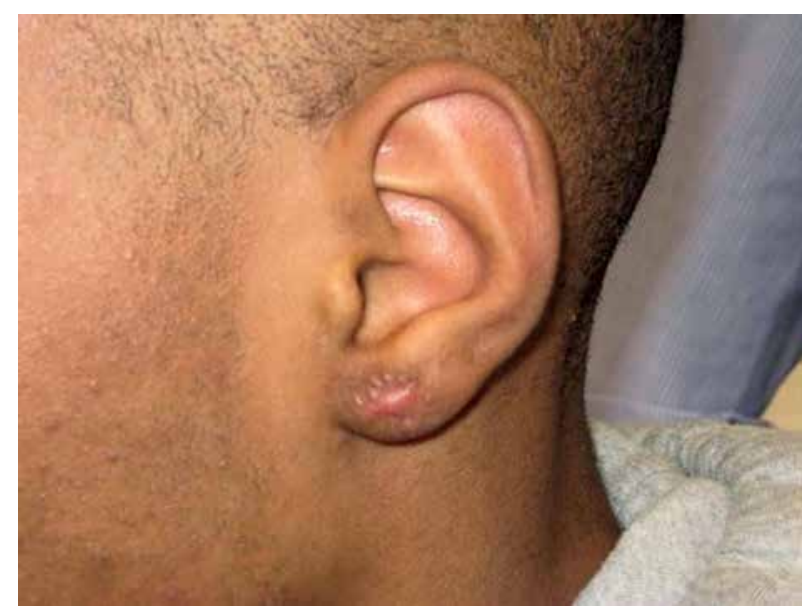

Fig. 8. Ear lobe keloid after the treatment

studies with a patient cohort and technique similar to the ones in this work.

There is no universally accepted radiation dose in the post-operative management of resected keloids, and the literature reports numerous dose and fractionation schedules [11]; however, the actual tendency to treat keloids is with low doses of radiotherapy without an increase in recurrence rate. In a study, Bijlard et al. described a lower risk of recurrence, with a BED as low as 19 Gy [4]. Guix et al. [8], in one of their articles with the highest keloid sample treated with HDR-BT, reported a control rate of $96.6 \%$ using a dose of 12 Gy in 4 fractions in 24 hours (BED, $15.6 \mathrm{~Gy}$ ), considering an $\alpha / \beta$ ratio of 10 . In Guix et al. series, the total dose was calculated $10 \mathrm{~mm}$ from the center of the source. Their results are slightly better than ours (recurrence rate of $4.4 \%$ vs. $4.9 \%$ ), but it is possible, as we discuss below, that the differences between the series are more in line with other factors, such as location of keloids in analyzed patients.

In our series, there is a trend for higher recurrence in the chest location, since $16 \%$ of keloids in this location have recurred. The locations distribution in the study of Guix et al. [8] differ from the locations of our study. The majority of keloids analyzed were located on the face. In our series, the most frequent location was the thorax. According to the published literature, the chest is often associated with higher recurrence rates $[12,13]$. Wagner et al. [12] and Ogawa et al. [14] also reported the chest as one of the locations with the highest recurrence rate. The differences were significant between treatment outcome and anatomical region of the keloids, with a recurrence rate of $49 \%(p<0.001)$

Although radiotherapy with surgery is considered as one of the most effective treatments for a benign pathology, such as keloids, there are groups of doctors who present various doubts about treating with radiotherapy for a risk of secondary malignancies. There is a lack of evidence about carcinogenic effects at low doses, which suggests two possibilities: the effects does not exist or they are too weak to appear as statistically significant, although in general, we can consider the reduced risk to produce a secondary tumor due to the treatment of keloids. In the published literature, the most import-

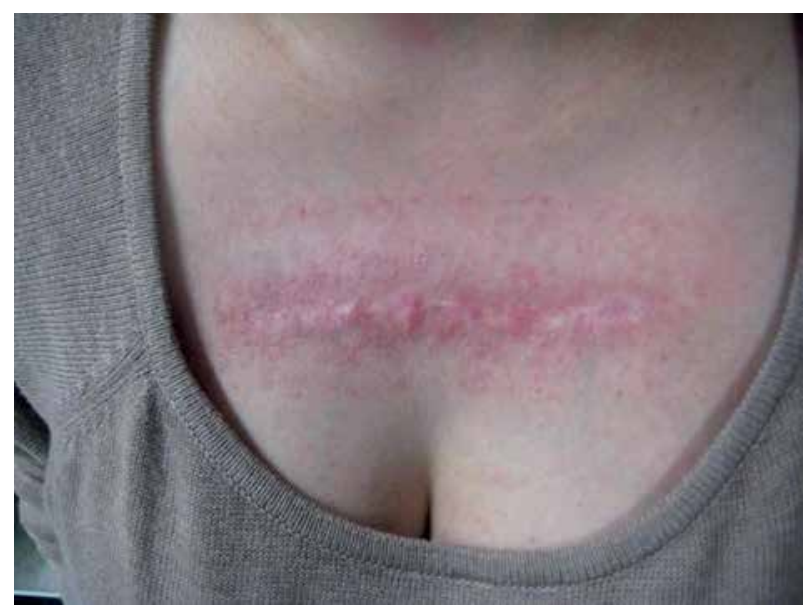

Fig. 9. Pre-sternal keloid after the treatment

ant risk factor is age at the time of exposure (higher in people who were young when exposed $<30$ years old) [16]. The patients analyzed in our study were older than 18 years old.

In our cohort, secondary malignancies that may be related to treatment with radiotherapy were not observed. However, it should be noted that our follow-up could not be considered as long enough to be able to observe them. The risk of carcinogenesis attributable to treatment with radiotherapy in keloid scars is very low, and acceptable for this treatment modality, but in places close to the thyroid gland and mammary glands, adequate protection should be performed [14].

\section{Conclusions}

In our series, high-dose-rate perioperative interstitial brachytherapy has proven to be a highly effective treatment, with a local failure rate of less than $5 \%$. All recurrences appeared in the thorax, one of the locations with the worst prognosis in the published literature, suggesting that a dose increase at this location may be necessary. The treatment was well tolerated by all patients, without presenting serious side effects. The cosmetic result was described as excellent/good in $81 \%$ of the patients.

\section{Acknowledgments}

The authors report no proprietary or commercial interest in any product mentioned or concept discussed in this article. This research did not receive any specific grant from funding agencies in the public, commercial, or not-for-profit sectors. The authors thank physic service of the Hospital Universitario de Asturias for the support in statistical analysis of the data in the project as well as the physicists in other centers: Ana Maria Barragán Montero (UCLouvain, Belgium) and Naipy Pérez (Innovative Cancer Center, Miami) for the review of the manuscript and valuable discussions.

\section{Disclosure}

The authors report no conflict of interest. 


\section{References}

1. Hellwege J, Russell S, Williams S et al. Gene-based evaluation of low frequency variation and genetically predicted gene expression impacting risk of keloid formation. Physiol Behav 2018; 176: 139-148.

2. Viani GA, Stefano EJ, Afonso SL et al. Postoperative strontium-90 brachytherapy in the prevention of keloids: results and prognostic factors. Int J Radiat Oncol Biol Phys 2009; 73: 1510-1516.

3. Goutos I, Ogawa R. Brachytherapy in the adjuvant management of keloid scars: literature review. Scars Burn Heal 2017; 3: 1-14.

4. Bijlard E, Verduijn GM, Harmeling JX et al. Optimal highdose-rate brachytherapy fractionation scheme after keloid excision; a retrospective multicenter comparison of recurrence rates and complications. Int J Radiat Oncol Biol Phys 2018; 100: 679-686.

5. Renz P, Hasan S, Gresswell S et al. Dose effect in adjuvant radiation therapy for the treatment of resected keloids. Int J Radiat Oncol 2018; 102: 149-154.

6. Borok TL, Bray M, Sinclair I et al. Role of ionizing irradiation for 393 keloids. Int J Radiat Oncol Biol Phys 1988; 15: 865-870.

7. Rockwell WB, Cohen IK, Ehrlich HP. Keloids and hypertrophic scars: A comprehensive review. Plast Reconstr Surg 1989; 84: 827-837.

8. Guix B, Henriquez I, Andres A et al. Treatment of keloids by high-dose-rate brachytherapy: a seven year study. Int J Radiat Oncol Biol Phys 2001; 50: 167-172.

9. Veen RE, Kal HB. Postoperative high-dose-rate brachytherapy in the prevention of keloids. Int J Radiat Oncol Biol Phys 2007; 69: 1205-1208.

10. Fowler JF. The linear-quadratic formula and progress in fractionated radiotherapy. Br J Radiol 1989; 62: 679-694.

11. Flickinger JC. A radiobiological analysis of multicenter data for postoperative keloid radiotherapy. Int J Radiat Oncol Biol Phys 2011; 79: 1164-1170.

12. Wagner W, Alfrink M, Micke O et al. Results of prophylactic irradiation in patients with resected keloids. A retrospective analysis. Acta Oncol 2000; 39: 217-220.

13. Guix B, Palma C. Irradiación intracavitaria con técnica de carga radiactiva diferida a control remoto. Tolerancia y complicaciones. Radiol 1998; 30: 430-437.

14. Ogawa R, Yoshitatsu S, Yoshida K et al. Is radiation therapy for keloids acceptable? The risk of radiation-induced carcinogenesis. Plast Reconstr Surg 2009; 124: 1196-1201.

15. Jiang P, Geenen M, Siebert FA et al. Efficacy and the toxicity of the interstitial high-dose-rate brachytherapy in the management of recurrent keloids: 5-year outcomes. Brachytherapy 2018; 17: 597-600.

16. Arenas M, Sabater S, Hernández V et al. Anti-inflammatory effects of low-dose radiotherapy. Strahlenther Onkol 2012; 188: 975-981. 